\title{
INFLUÊNCIA DE DIFERENTES ADESIVOS UTILIZADOS NA FABRICAÇÃO DE PAINÉIS AGLOMERADOS NA AÇÃO DE Coptotermes gestroi (WASMANN, 1896) (ISOPTERA: RHINOTERMITIDAE)
}

\author{
Flávia Cristiana da Silva ${ }^{1}$; Henrique Trevisan ${ }^{2}$; Kleber Eduardo Dias Silva ${ }^{3}$; Roberto Carlos Costa Lelis ${ }^{4}$; \\ Acácio Geraldo de Carvalho ${ }^{4}$ \\ ${ }^{1}$ Graduanda em Engenharia Florestal, UFRRJ, Seropédica, RJ, Brasil - flaviacristiana@ bol.com.br \\ ${ }^{2}$ Eng. Florestal, Doutorando em Ciências Florestais, UFRRJ, Seropédica, RJ, Brasil - trevisan@ufrrj.br \\ ${ }^{3}$ Eng. Florestal, Mestrando em Ciências Florestais, UFRRJ, Seropédica, RJ, Brasil \\ ${ }^{4}$ Eng. Florestal, Dr., Depto. de Produtos Florestais, UFRRJ, Seropédica, RJ, Brasil - lelis@ufrrj.br - acacio@ufrrj.br
}

Recebido para publicação: 07/04/2009 - Aceito para publicação: 07/08/2009

\begin{abstract}
Resumo
O objetivo deste trabalho foi avaliar a resistência de painéis aglomerados à ação de Coptotermes gestroi (WASMANN, 1896). Os painéis aglomerados foram confeccionados com partículas provindas de Pinus caribaea var. caribaea, utilizando-se resinas tânicas, produzidas a partir de tanino da casca de Eucalyptus pellita e de Acacia mearnsii. A metodologia utilizada consistiu em atrair os térmitas para uma câmara de alvenaria, sem interferir na colônia, usando como isca placas de papelão. Foram distribuídos corpos de prova medindo 5,0 x 2,5 x 1,27 cm, expostos ao ataque durante noventa dias. Após esse período, foram realizadas avaliações nos corpos de prova. Foi constatado que as amostras que continham fenol-formaldeído apresentaram-se mais resistentes à ação de $C$. gestroi, enquanto que as amostras que continham tanino-formaldeído de Acacia mearnsii, ou a combinação de taninoformaldeído de Acacia mearnsii e Eucalyptus pellita, mostraram-se menos resistentes à ação do térmita.

Palavras-chave: Tanino; painéis de aglomerados; térmita.
\end{abstract}

\begin{abstract}
Influence of different adhesive utilized for manufacture of particleboards, under activity by Coptotermes gestroi (Wasmann, 1896) (Isoptera: Rhinotermitidae). The objective of this work was to study the resistance of particleboards under activity by Coptotermes gestroi (WASMANN, 1896). The particleboards were made with particles originating from Pinus caribaea var. caribaea, using tannin resin, produced from tannin of Eucalyptus pellita and Acacia mearnsii (De Wild). The methodology used consists on attracting termites to masonry camera, without interfering in the colony, using cardboard as bait. Samples of $5,0 \times 2,5 \times 1,27 \mathrm{~cm}$ were exposed to the attack of the same ones for ninety days. After that period, evaluations were accomplished for verification the performance of them on the samples. It was verified that the samples which contained fenolformaldeído, be came more resistant to the action of the termite and the ones that contained tannin of Acacia mearnsii, or tannin of Acacia mearnsii and Eucalyptus pellita, are less resistant to the action of these termite.

Keywords: Tannin; particleboards; termite.
\end{abstract}

\section{INTRODUÇÃO}

Chapas de aglomerado são painéis de madeira reconstituída formados a partir da redução da madeira em partículas, que são impregnadas com resina e arranjadas formando um colchão. Esse colchão, pela ação controlada de calor, pressão e umidade, adquire uma forma definitiva e estável. Essas chapas são comumente utilizadas na fabricação de móveis, forros, embalagens etc.

Segundo Gonçalves et al. (2003), nos últimos anos vários materiais têm sido pesquisados para substituir as resinas sintéticas na fabricação desses painéis, com destaque para o tanino, polifenol obtido 
de várias fontes renováveis, como, por exemplo, da casca de Acacia mearnsii, pínus e da madeira do cerne de quebracho. Os adesivos à base de taninos são denominados tanino-formaldeídos (TF), e são obtidos pela reação de flavonoides poliméricos naturais (taninos condensados) com formaldeído (Pizzi; Mittal, 1994).

A maioria das pesquisas versa sobre a avaliação dos aspectos tecnológicos desses painéis, sendo, portanto, parcas as pesquisas sobre a influência dessas resinas na durabilidade dessas peças, frente à ação de organismos decompositores. Nesse sentido, Okino et al. (2007) relatam que o conhecimento da resistência de painéis à base de madeira reconstituída ao ataque de micro-organismos deve servir de base para a prevenção da degradação e a correta destinação para uso do material. Relatam que o uso de espécies madeireiras naturalmente mais resistentes ao ataque de micro-organismos é mais vantajoso do que a utilização de produtos químicos preservativos na confecção de painéis de aglomerado. Portanto, esse raciocínio pode ser estendido às resinas utilizadas na fabricação dos painéis, sendo vantajosa, também, a adoção de resinas que tenham a propriedade de conferir resistência às chapas frente à atuação dos organismos xilófagos.

Em peças que não estão em contato direto com o solo, têm-se como maiores agentes deterioradores os térmitas. Embora seja fato que existam menos trabalhos com térmitas em comparação com fungos xilófagos, Silva et al. (2004) afirmam que pesquisadores brasileiros e de outras partes do mundo têm-se preocupado com o problema dos cupins, criando, portanto, novas linhas de pesquisa nessa área.

O objetivo deste trabalho foi avaliar a influência de diferentes resinas, entre elas as tânicas, utilizadas na fabricação de painéis aglomerados, na ação do Coptotermes gestroi.

\section{MATERIAL E MÉTODOS}

Os painéis de aglomerados, dos quais foram extraídos os corpos de prova, foram fabricados no Laboratório de Painéis de Madeira, Departamento de Produtos Florestais (DPF) da UFRRJ, com partículas de madeira de Pinus caribaea var. caribaea, proveniente de plantios localizados na UFRRJ.

As resinas utilizadas foram à base de tanino-formaldeído de Acacia mearnsii e Ecalyptus pellita e fenol-formaldeído, com respectivos teores e tempos de prensagem expressos na tabela 1 . O experimento constituiu-se de 13 tratamentos, com seis repeticões cada. A testemunha foi constituída de madeira de pinus, de conhecida baixa durabilidade.

Tabela 1. Descrição dos tratamentos para confecção dos painéis de aglomerados.

Table 1. Description of the treatments for manufacture of particleboards.

\begin{tabular}{lcc}
\hline Tratamento & Adesivo & T.P. $($ min.) \\
\hline T1 & Tanino-formaldeído (TF) de Acacia mearnsii a 45\% & 6 \\
T2 & Tanino-formaldeído (TF) de Acacia mearnsii a 45\% & 9 \\
T3 & Fenol-formaldeído (FF) & 6 \\
T4 & Fenol-formaldeído (FF) & 9 \\
T5 & FF + TF de Acacia mearnsii a 45\% (90:10) & 6 \\
T6 & FF + TF de Acacia mearnsii a 45\% (90:10) & 9 \\
T7 & FF + TF de Acacia mearnsii a 45\% (80:20) & 6 \\
T8 & FF + TF de Acacia mearnsii a 45\% (80:20) & 9 \\
T9 & TF (Acacia mearnsii + E. pellita ) a 45\% (90:10) & 6 \\
T10 & TF (Acacia mearnsii + E. pellita) a 45\% (90:10) & 9 \\
T11 & TF (Acacia mearnsii + E. pellita) a 45\% (80:20) & 6 \\
T12 & TF (Acacia mearnsii + E. pellita) a 45\% (80:20) & 9 \\
T13 & Madeira de Pinus caribaea var. caribaea & - \\
\hline
\end{tabular}

T.P.: Tempo de prensagem em minutos.

Os corpos de prova, nas dimensões de 5,0 x 2,5 x 1,27 cm, foram mantidos em sala de climatização (temperatura de $20 \pm 1{ }^{0} \mathrm{C}$ e umidade relativa de $65 \pm 3 \%$ ), para estabilização da umidade. Após atingirem a umidade de equilíbrio, foram determinados volume e massa desses corpos, para determinação da densidade aparente, baseando-se na relação da massa pelo volume. Inicialmente, as amostras foram secas em estufa à temperatura de $105 \pm 3{ }^{\circ} \mathrm{C}$ por 24 horas, para obtenção da massa seca. 
Foi utilizada uma colônia de cupim da espécie $C$. gestroi ocorrente naturalmente no campus da UFRRJ. Para a montagem dos testes, os insetos foram atraídos para o interior de uma câmara escura, construída com elementos de alvenaria, com dimensões de 80 × 80 x $80 \mathrm{~cm}$, utilizando-se papelão como atrativo. Este foi acomodado em toda a base da estrutura, servindo também para uniformizar a ocorrência dos térmitas dentro dela, seguindo recomendações de Trevisan (2006). Após esse processo, com cerca de $90 \%$ do papelão consumido, os corpos de prova foram distribuídos dentro da câmara em blocos ao acaso, contendo seis blocos com treze amostras cada.

$\mathrm{O}$ acesso ao interior da estrutura foi fechado com uma placa de isopor e só foi aberto após 90 dias. Segundo Abreu; Silva (2000), 45 dias seria tempo suficiente para que a madeira de pinus utilizada como testemunha perdesse quase toda a sua massa, porém esse tempo não foi insuficiente para o consumo total da madeira da testemunha, motivo pelo qual decidiu-se utilizar um tempo de exposição maior (90 dias).

Para a avaliação do desgaste dos corpos de prova causado pelos cupins, utilizou-se tabela sugerida pela norma ASTM D-3345 (1994), pela qual atribuem-se notas aos corpos de prova pelo dano que lhes foi causado (Tabela 2). Também foi utilizado o sistema de classificação de durabilidade sugerido por Abreu; Silva (2000) (Tabela 3). Foi utilizado o teste de Tukey a um nível de significância de 5\%, para comparação das médias.

Tabela 2. Avaliação do desgaste provocado pelos cupins nos corpos de prova.

Table 2. Evaluation of the damage by termites stired up of in the samples.

\begin{tabular}{lc}
\hline Tipos de desgaste & Nota \\
\hline Sadio, permitindo escarificações superficiais & 10 \\
Ataque superficial & 9 \\
Ataque moderado, havendo penetração & 7 \\
Ataque intensivo & 4 \\
Falha, havendo ruptura dos corpos de prova & 0 \\
\hline
\end{tabular}

Tabela 3. Avaliação da durabilidade em função da perda percentual de massa dos corpos de prova expostos ao ataque natural de Coptotermes gestroi.

Table 3. Evaluation of durability, as function of loss perceptual mass at the samples.

\begin{tabular}{lc}
\hline Perda de massa $(\%)$ & Classe \\
\hline 0 a 10 & Altamente resistente (AR) \\
11 a 24 & Resistente (R) \\
Acima de 24 & Não resistente (NR) \\
\hline
\end{tabular}

\section{RESULTADOS E DISCUSSÃO}

Todas as comparações estatísticas entre os valores médios da densidade aparente dos corpos de prova confeccionados com painéis de aglomerado com diferentes resinas e tempos de prensagem não foram diferentes estatisticamente (Tabela 4). Esses valores, quando comparados com o valor médio da densidade da testemunha, apresentaram-se diferentes estatisticamente, excetuando-se as comparações realizadas com a densidade média dos corpos de prova do tratamento 7 , sendo este valor igual, estatisticamente, à média da testemunha, madeira de pinus, pelo teste de Tukey a 5\% de significância (Tabela 4).

Os resultados indicados na tabela 4 mostram que a densidade dos painéis não apresentou influência direta no ataque por $C$. gestroi aos corpos de prova, pois, apesar da testemunha, composta de madeira de pinus (T13), apresentar a menor densidade em relação aos demais tratamentos, ela também apresentou valores inferiores de consumo médio em gramas e perda de massa percentual, em comparação com os corpos de prova classificados como não resistentes (NR) (Tabela 4).

Os menores valores de consumo médio, em gramas, foram obtidos da avaliação dos corpos de prova manufaturados com chapas de aglomerado que utilizaram as resinas à base de fenol-formaldeído (FF) - T3, T4 -, e à base de fenol-formaldeído + tanino-formaldeído (FF+TF) - T5, T6, T7 e T8 -, sendo os respectivos valores médios $(0,31,0,67,0,77,0,74,0,50$ e 0,80$)$ iguais, estatisticamente, pelo teste de Tukey a 5\% de significância (Tabela 4). Já os maiores valores de consumo médio, em gramas, foram dos corpos de prova manufaturados com chapas de aglomerado que utilizaram as resinas à base de taninoformaldeído (TF): T1, T2, T9, T10, T11 e T12, além da testemunha (T13). 
Tabela 4. Densidade aparente média $( \pm \mathrm{DP})$ em $\mathrm{g} / \mathrm{cm}^{3}$, consumo médio $( \pm \mathrm{DP})$ em gramas, desgaste médio, perda de massa percentual média $( \pm \mathrm{DP})$ e classe de resistência dos corpos de prova de aglomerados expostos ao ataque natural de Coptotermes gestroi pelo período de 90 dias.

Table 4. Density apparent medium $( \pm \mathrm{DP})$ in $\mathrm{g} / \mathrm{cm}^{3}$, consuming medium $( \pm \mathrm{DP})$ in gramma, consuming medium, loss of mass perceptual medium $( \pm \mathrm{DP})$ and order of resistance of the samples at particleboards, exposed to the attack for ninety days of the Coptotermes gestroi.

\begin{tabular}{|c|c|c|c|c|c|}
\hline Trat. & $\begin{array}{c}\text { Densidade } \\
\left(\mathrm{g} / \mathrm{cm}^{3}\right)\end{array}$ & Consumo médio (g) & Desgaste médio & $\begin{array}{c}\text { Perda de massa } \\
(\%)\end{array}$ & $\begin{array}{c}\text { Classe de } \\
\text { resistência }\end{array}$ \\
\hline T1 & $0,60 \pm 0,030^{\mathrm{a}}$ & $6,98 \pm 0,49^{\mathrm{ab}}$ & 0,00 & $94,68 \pm 4,81^{\text {a }}$ & $\mathrm{NR}$ \\
\hline $\mathrm{T} 2$ & $0,66 \pm 0,023^{\mathrm{a}}$ & $7,39 \pm 0,36^{\mathrm{a}}$ & 0,00 & $95,03 \pm 4,72^{\mathrm{a}}$ & NR \\
\hline $\mathrm{T} 3$ & $0,65 \pm 0,015^{\text {a }}$ & $0,31 \pm 0,14^{\mathrm{c}}$ & 9,67 & $4,11 \pm 1,90^{\mathrm{b}}$ & $\mathrm{AR}$ \\
\hline $\mathrm{T} 4$ & $0,64 \pm 0,071^{\text {a }}$ & $0,67 \pm 0,29^{\mathrm{c}}$ & 9,50 & $9,05 \pm 4,97^{\mathrm{b}}$ & AR \\
\hline T5 & $0,65 \pm 0,095^{\mathrm{a}}$ & $0,77 \pm 0,60^{c}$ & 8,50 & $10,64 \pm 9,03^{b}$ & $\mathrm{R}$ \\
\hline T6 & $0,65 \pm 0,040^{a}$ & $0,74 \pm 0,69^{c}$ & 8,67 & $9,85 \pm 9,93^{b}$ & AR \\
\hline $\mathrm{T} 7$ & $0,53 \pm 0,261$ ab & $0,50 \pm 0,16^{\mathrm{c}}$ & 8,17 & $6,55 \pm 2,36^{\mathrm{b}}$ & $\mathrm{AR}$ \\
\hline $\mathrm{T} 8$ & $0,66 \pm 0,051^{\mathrm{a}}$ & $0,80 \pm 0,58^{\mathrm{c}}$ & 8,83 & $10,49 \pm 8,19^{\mathrm{b}}$ & $\mathrm{R}$ \\
\hline T9 & $0,61 \pm 0,026^{\mathrm{a}}$ & $7,34 \pm 0,21^{\mathrm{a}}$ & 0,00 & $92,66 \pm 0,21^{\text {a }}$ & NR \\
\hline $\mathrm{T} 10$ & $0,61 \pm 0,044^{\mathrm{a}}$ & $7,35 \pm 0,42^{a}$ & 0,00 & $92,65 \pm 0,42^{\mathrm{a}}$ & NR \\
\hline T11 & $0,63 \pm 0,030^{\mathrm{a}}$ & $7,1 \pm 0,34^{\mathrm{a}}$ & 0,00 & $92,91 \pm 0,34^{\mathrm{a}}$ & NR \\
\hline $\mathrm{T} 12$ & $0,62 \pm 0,030^{\text {a }}$ & $7,08 \pm 0,40^{a}$ & 0,00 & $97,39 \pm 1,73^{\text {a }}$ & NR \\
\hline $\mathrm{T} 13$ & $0,43 \pm 0,030^{\mathrm{b}}$ & $6,22 \pm 0,43^{\mathrm{b}}$ & 0,00 & $95,12 \pm 2,86^{\mathrm{a}}$ & NR \\
\hline
\end{tabular}

T1: TF de acácia $45 \%$ a 6 min.; T2: TF de acácia $45 \%$ a 9 min.; T3: FF a 6 min.; T4: FF a 9 min.; T5: FF + TF de acácia 45\% (90:10) a 6 min.; T6: FF + TF de acácia 45\% (90:10) a 9 min.; T7: FF + TF de acácia 45\% (80:20) a 6 min.; T8: FF + TF de acácia 45\% (80:20) a 9 min.; T9: TF de acácia 45\% + TF de eucalipto a 45\% (90:10) a 6 min.; T10: TF de acácia 45\% + TF de eucalipto a 45\% (90:10) a 9 min.; T11: TF de acácia 45\% + TF de eucalipto a 45\% (80:20) a 6 min.; T12: TF de acácia 45\% + TF de eucalipto a 45\% (80:20) a 9 min.; T13: Madeira de pinus; AR = Altamente resistente; $\mathrm{R}=\mathrm{Resistente}$; NR = Não resistente. Letras iguais não diferem ente si, ao nível de $5 \%$ de significância, pelo teste de Tukey.

Os dados de desgaste médio indicam o estado sanitário dos corpos de prova, e os resultados revelaram que os corpos de prova manufaturados com chapas de aglomerado que utilizaram as resinas à base de FF (T3, T4) e FF+TF (T5, T6, T7 e T8) foram os mais resistentes ao desgaste pelos térmitas, apresentando as seguintes notas, respectivamente: 9,$67 ; 9,50 ; 8,50 ; 8,67 ; 8,17$ e 8,83. Os corpos de prova fabricados com chapas de aglomerado que utilizaram as resinas à base de TF (T1, T2, T9, T10, T11 e T12), além da testemunha (T13), apresentaram nota zero, indicando que houve ruptura dos corpos de prova.

Uma análise desse resultado sugere que resinas formuladas somente com $\mathrm{TF}_{\mathrm{acácia}}$ ou $\mathrm{TF}_{\text {acáciateucalipto }}$ utilizadas na fabricação de chapas de aglomerados conferem uma baixa resistência aos painéis ao ataque de C. gestroi. É importante destacar que todos os corpos de prova manufaturados com chapas de aglomerado que utilizaram apenas resinas à base de tanino-formaldeído (TF), como os tratamentos T1, T2, T9, T10, T11 e T12, apresentaram os maiores consumos médios, em gramas, os maiores desgastes, as maiores perdas de massa percentual, e foram classificados como não resistentes (Tabela 4).

Os corpos de prova fabricados com painéis de aglomerado que utilizaram as resinas à base das combinações FF+TF (T5, T6, T7 e T8) mostraram-se tão resistentes quanto os corpos de prova manufaturados com painéis de aglomerado que utilizaram resinas somente à base de fenol-formaldeído (FF), como T3 e T4. Quando os valores médios do consumo e da perda de massa percentual desses tratamentos foram comparados pelo teste de Tukey a um nível de significância de 5\%, demonstraram-se iguais. Isso indica que a combinação de $\mathrm{FF}+\mathrm{TF}$ pode conferir resistência a $C$. gestroi, em diferentes teores.

Já para os corpos de prova que foram provenientes de painéis de aglomerado manufaturados exclusivamente com TF de A. mearnsii + E. pellita, em diferentes proporções, os resultados confirmaram que essas resinas conferem baixa resistência à ação de C. gestroi, como observado em T1, T2, T9, T10, T11, T12 e T13. Esses resultados podem ser evidenciados com maior clareza no gráfico que indica perda de massa percentual por tratamento (Figura 1). 
Segundo o sistema de classificação de durabilidade sugerido por Abreu; Silva (2000), os corpos de prova fabricados com painéis de aglomerado que utilizaram as resinas FF a 6 min. (T3), FF a 9 min. (T4), FF+TF de acácia 45\% (90:10) a 9 min. (T6) e FF+TF de acácia 45\% (80:20) a 6 min. (T7) apresentaram-se altamente resistentes (AR) ao ataque de $C$. gestroi.

Já os corpos de prova fabricados com painéis de aglomerado que utilizaram as resinas FF+TF de acácia 45\% (90:10) a 6 min. (T5) e FF+TF de acácia 45\% (80:20) a 9 min. (T8) foram classificados como resistentes (R) ao ataque de $C$. gestroi.

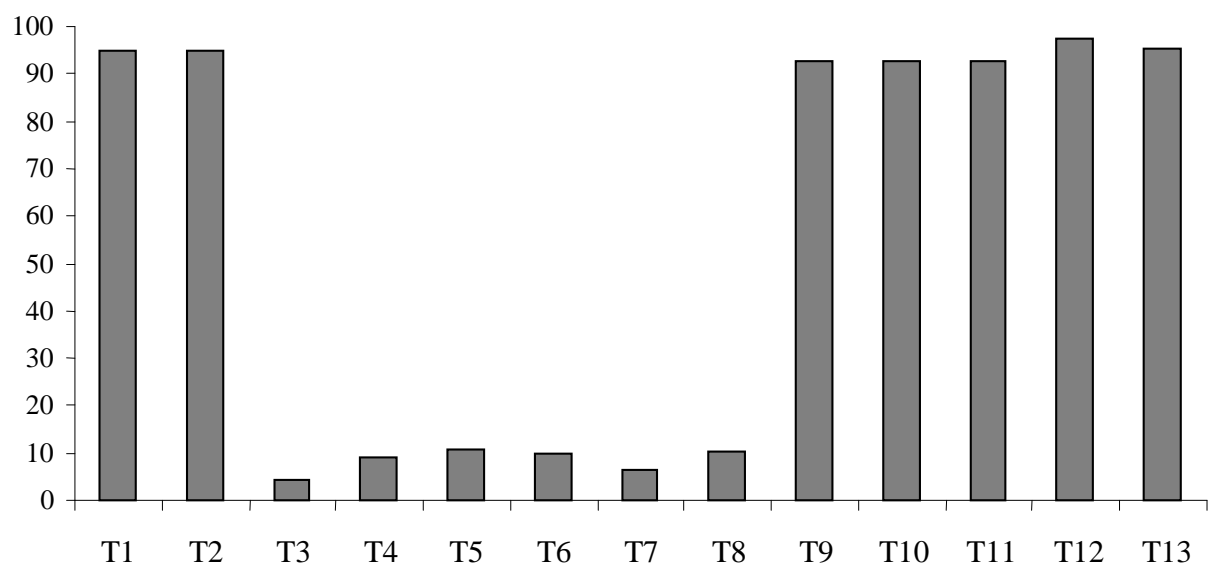

Figura 1. Perda de massa percentual por tratamento dos corpos de prova fabricados com diferentes tipos de resina e submetidos à ação de Coptotermes gestroi por 90 dias.

Figure 1. Perceptual mass loss by treatment, at the samples manufactured with different type of resin under the action of termite for ninety days.

Portanto, os corpos de prova fabricados com painéis de aglomerado que utilizaram as resinas TF de acácia $45 \%$ a 6 min. (T1), TF de acácia $45 \%$ a 9 min. (T2), TF de acácia + eucalipto 45\% (90:10) a 6 min . (T9), TF de acácia + eucalipto 45\% (90:10) a 9 min. (T10), TF de acácia + eucalipto 45\% (80:20) a 6 min. (T11), TF de acácia + eucalipto 45\% (80:20) a 9 min. (T12), além da testemunha de madeira de pinus (T13), foram classificados como não resistentes (NR) ao ataque de $C$. gestroi.

Em relação à ação de taninos em insetos Ayres et al. (1997) verificaram que a rápida mortalidade de insetos tratados com taninos condensados parece ser devido à atividade tóxica desses compostos, e não pela inibição da digestibilidade. Lunz et al. (2007), associando a ocorrência de coleópteros xilófagos com o teor de tanino na madeira de três leguminosas, concluíram que a espécie arbórea que apresentou o menor teor de tanino teve uma menor incidência desse grupo de insetos.

Dessa maneira, pode-se inferir que adesivos à base de resinas tânicas poderiam conferir certa resistência à ação dos térmitas, em face do seu efeito tóxico a insetos, no entanto isso não ocorreu. Dessa forma, pode-se indicar que os taninos utilizados na fabricação das resinas não apresentam atividade inseticida a essa espécie de térmita, ou mesmo que o processo de fabricação das resinas e chapas possa interferir nas propriedades inseticidas do tanino. Logo, essas inferências sugerem novas linhas de pesquisas para elucidar essas questões.

\section{CONCLUSÕES}

- Os diferentes tempos de prensagem não influenciaram na ação de Coptotermes gestroi.

- Os painéis de aglomerado produzidos somente com resinas à base de tanino-formaldeído de acácianegra ou à base de tanino-formaldeído de acácia-negra e eucalipto apresentaram-se mais suscetíveis à ação de Coptotermes gestroi.

- Os painéis de aglomerado produzidos somente com resinas à base de fenol-formaldeído são mais resistentes à ação de Coptotermes gestroi. 
- Os painéis de aglomerados que utilizaram a combinação das resinas à base de fenol-formaldeído (FF) modificado com tanino-formaldeído (TF) de acácia-negra, nas proporções 90:10 e 80:20, são resistentes à ação de Coptotermes gestroi.

- As formulações das resinas à base de tanino-formaldeído apresentaram baixa resistência à ação de Coptotermes gestroi, porém foram utilizadas sem adição de qualquer tipo de preservativo.

- O método de "semicampo" utilizado neste trabalho mostrou-se eficaz para a avaliação da ação de Coptotermes gestroi em painéis de aglomerados, por não haver interferência na colônia e por respeitar as características ecológicas dos térmitas.

- Novos estudos devem ser implementados a fim de verificar quais tipos de taninos teriam toxicidade ou ação repelente a esse térmita em particular, de modo a se analisar sua ação em adesivos para painéis manufaturados.

\section{REFERÊNCIAS}

ABREU, R. L. S.; SILVA, K. E. S. Resistência natural de dez espécies madeireiras da Amazônia ao ataque de Nasutitermes macrocephalus (Silvestri) e N. surinamensis (Holmgrem) (Isoptera: Termitidae). Revista Árvore, Viçosa, v. 24, n. 2, p. 229-234, 2000.

AMERICAN SOCIETY FOR TESTING AND MATERIALS - ASTM D- 3345. Standard method for laboratory evaluation of wood and other cellulosic materials for resistente to termites. Annual Book of ASTM Standards, Philadelphia, v. 0410, p. 439-41, 1994.

AYRES, M. P.; CLAUSEN, S. F.; MACLEAN, JR.; REDMAN, A. M.; REICHARDT, P. B. Diversity of structure and antiherbivore activity in condensed tannins. Ecology, v. 78: p. 1696-1712, 1997.

GONÇALVES, C. A.; LELIS, R. C. C.; BRITO, E. O.; NASCIMENTO, A. M. Produção de chapas de madeira aglomerada com adesivo ureia-formaldeído modificado com tanino de Mimosa caesalpiniaefolia Bentham (sabiá). Floresta e Ambiente, Seropédica-RJ, v. 10, n. 1, p. 18-26, 2003.

LUNZ, A. M.; CARVALHO, A. G.; JORGE, A. C.; CARVALHO, M. G.; GOMES, M. S. R. Prospecção toquímica de três leguminosas e associação com incidência de coleópteros xilófagos. Floresta e Ambiente, Seropédica-RJ, v. 14, n. 1, p. 06-13, 2007.

OKINO, E. Y. A.; ALVES, M. V. S.; TEIXEIRA, D. E.; SOUZA, M. R.; SANTANA, A. E. Biodegradação de chapas de partículas orientadas de pinus, eucalipto e cipreste expostas a quatro fungos apodrecedores. Scientia Forestalis. n. 74, p. 67-74, 2007.

SILVA, J. C.; CABALLEIRA LOPEZ, A. G.; OLIVEIRA, J. T. S. Influência da idade na resistência natural da madeira de Eucalyptus grandis W. Hill ex. Maiden ao ataque de cupim de madeira seca (Cryptotermes brevis). Revista Árvore, v. 28, n. 4, p. 583-587, 2004.

TREVISAN, H. Degradação natural de toras e sua influência na madeira de cinco espécies florestais. Dissertação (Mestrado em Ciências Ambientais e Florestais) UFRRJ. IF, 2006. 56 p. 